\title{
Spontaneous Baryogenesis in Supersymmetric Models
}

\author{
S. A. Abel ${ }^{1}$, W. N. Cottingham ${ }^{2}$ and I. B. Whittingham ${ }^{3}$ \\ ${ }^{1}$ Rutherford Appleton Laboratory \\ Chilton, Didcot \\ Oxon OX11 0QX \\ England
}

${ }^{2}$ H. H. Wills Physics Laboratory
Royal Fort, Tyndal Avenue
Bristol BS8 1TL
England

${ }^{3}$ Department of Physics

James Cook University

Townsville

Australia 4811

September 19, 2018

Abstract

\begin{abstract}
In this paper we extend the results of previous work on spontaneous baryogenesis to general models involving CP violation in the Higgs sector. We show how to deal with Chern-Simons terms appearing in the effective potential arising from phase changes in the vacuum expectation values of the Higgs fields. In particular, this enables us to apply this mechanism to general supersymmetric models including the minimal supersymmetric standard model, and the extended model with a gauge singlet. A comparison is made between this approach, and that in which one solves the equations of motion for Higgs winding modes. As anticipated in earlier work, the effect of the latter approach is found to be small.
\end{abstract}




\section{Introduction}

There has been considerable recent interest [1]-[11] in the possibility of generating the observed matter-antimatter asymmetry of the universe at the electroweak phase transition within the standard electroweak model or its minimal extension to include an additional Higgs doublet (for recent reviews see Ref. [12]). It has been well known since the pioneering work of t' Hooft [13] that, due to the axial anomaly, baryon number $B$ and lepton number $L$ are not conserved in the standard model. For $N_{g}$ fermion generations the change in baryon and lepton number is given by

$$
\Delta B=\Delta L=N_{g} \Delta N_{C S}
$$

where $N_{C S}$, the Chern-Simons number for the gauge field, is given by

$$
N_{C S}=\int d^{3} x K^{0}
$$

with $K^{\mu}$ the topological current

$$
K^{\mu}=\frac{g_{2}^{2}}{16 \pi^{2}} \varepsilon^{\mu \nu \rho \lambda} \operatorname{Tr}\left(F_{\nu \rho} A_{\lambda}+\frac{2}{3} i g_{2} A_{\nu} A_{\rho} A_{\lambda}\right)
$$

and $A_{\mu}=A_{\mu}^{a} \sigma^{a} / 2, F_{\mu \nu}=F_{\mu \nu}^{a} \sigma^{a} / 2$ the $S U(2)$ gauge field and field strength respectively. In Eq.(1) the change in $N_{C S}$ is associated with transitions between the different topological sectors of the gauge and Higgs fields. The different sectors are separated by energy barriers corresponding to the saddle point sphaleron configuration of mass $M_{s p h} \approx 5 M_{W} / \alpha_{W}$ (where $\alpha_{W}=g_{2}^{2} / 4 \pi$ ) so that these transitions are heavily suppressed at zero temperature where $M_{s p h} \approx 10 \mathrm{TeV}$ and the $\Delta N_{C S}=1$ transitions are mediated by quantum instanton tunneling at the rate $\exp \left(-4 \pi / \alpha_{W}\right)$. However at high temperatures the transitions can occur rapidly via classical transitions induced by thermal fluctuations over the now lower energy sphaleron.

The effective action for the gauge and Higgs fields at temperature $T$ contains the CP-violating Chern-Simons term [2]

$$
\Delta S_{C P} \propto \int d^{4} x f(\phi, T) F^{\mu \nu} \tilde{F}_{\mu \nu}
$$

where $\tilde{F}^{\mu \nu}=\varepsilon^{\mu \nu \rho \lambda} F_{\rho \lambda}$, and $f(\phi, T)$ is a gauge invariant function constructed from the Higgs doublets $\phi$ of the model. This term is heavily suppressed in the standard model, being of order $10^{-20}$ [14. However it has been argued [2, 4] that it may be much larger in multi-Higgs doublet models with soft CP-violation in the Higgs sector. Thus these extended electroweak models meet two of Sakharov's [15] conditions for generation of a matter-antimatter asymmetry, that is the existence of (anomalous) $B$ violation and of $C P$ violation. The third condition, the departure from thermal equilibrium, may occur during the electroweak phase transition if it is first order [10, 12, 16, 17].

An interesting explicit scenario for the generation of a baryonic asymmetry has been suggested [2, 4, 7, 8] in which the Chern-Simons term (41), arising from soft CP-violating terms in the Higgs potential, has the form of a CP-odd Higgs phase linearly coupled to $N_{C S}$ and modifies the classical equations of motion for the gauge fields such that, during the electroweak phase transition, an asymmetry is produced in the probabilities of the topology changing processes associated with $\Delta N_{C S}=+1$ and -1 , thereby driving the $N_{C S}$ value of the universe positive.

An alternative scenario has been advocated by Cohen et al [1, 5, 6] in which the physical pseudoscalar field $\theta(x)$ orthogonal to the Goldstone boson, develops a derivative coupling to the fermionic hypercharge current $j_{Y}^{\mu}$

$$
\delta \mathcal{L}=2 \partial_{\mu} \theta j_{Y}^{\mu}
$$


as a result of performing an anomaly-free space-time dependent rotation on the fermion fields to remove $\theta(x)$ from the Yukawa couplings. The spatial average of $\dot{\theta}$ acquires a non-zero value during the electroweak phase transition and acts as a potential for fermionic hypercharge which produces a free energy which is minimised for non-zero baryon number. This mechanism requires a first order phase transition, in order that the produced baryon asymmetry be preserved against sphaleron transitions, which for the standard model and MSSM translates into a stringent bound on the physical Higgs masses $\left(m_{H}<55 \mathrm{GeV}\right.$ [10], and $m_{H}<64 \mathrm{GeV}$ [11] respectively).

In general it is possible to only couple $\dot{\theta}$ to hypercharge, when only one Higgs is responsible for the fermion masses. Because of this, the mechanism has not yet been applied explicitly to supersymmetric models. In this paper we examine the relation between these two effects. First we extend the results of Ref. [5] to the most general case. We then rederive the main result of Ref.[2], using the rotated-phase approach of Ref.[5], and find this effect to be negligible compared with the former as anticipated in earlier work. We conclude by applying the results to the minimal supersymmetric standard model (MSSM) and the extended model with gauge singlet (ESSM).

\section{The two-Higgs model}

In order to clarify the connection between the approaches described above, we consider the most general soft CP-violating, two Higgs doublet model [18]. As in the supersymmetric Standard Model, one doublet field, $\phi_{1}$, gives mass to the up-type quarks and the other, $\phi_{2}$, to the downtype quarks and charged leptons. The zero temperature Higgs potential is

$$
\begin{aligned}
V_{0}= & \lambda_{1}\left(\phi_{1}^{\dagger} \phi_{1}-v_{1}^{2}\right)^{2}+\lambda_{2}\left(\phi_{2}^{\dagger} \phi_{2}-v_{2}^{2}\right)^{2} \\
& +\lambda_{3}\left(\phi_{1}^{\dagger} \phi_{1}-v_{1}^{2}+\phi_{2}^{\dagger} \phi_{2}-v_{2}^{2}\right)^{2} \\
& +\lambda_{4}\left[\left(\phi_{1}^{\dagger} \phi_{1}\right)\left(\phi_{2}^{\dagger} \phi_{2}\right)-\left(\phi_{1}^{\dagger} \phi_{2}\right)\left(\phi_{2}^{\dagger} \phi_{1}\right)\right] \\
& +\lambda_{5}\left[\operatorname{Re}\left(\phi_{1}^{\dagger} \phi_{2}\right)-v_{1} v_{2} \cos \xi\right]^{2} \\
& +\lambda_{6}\left[\operatorname{Im}\left(\phi_{1}^{\dagger} \phi_{2}\right)-v_{1} v_{2} \sin \xi\right]^{2}
\end{aligned}
$$

With suitable values of the parameters $\lambda_{k}$ (all positive for example), this potential is minimised for fields, up to $S U(2) \times U(1)$ gauge transformations, of the form

$$
\left\langle\phi_{1}\right\rangle=\left(\begin{array}{c}
0 \\
v_{1}
\end{array}\right) \quad\left\langle\phi_{2}\right\rangle=\left(\begin{array}{c}
0 \\
v_{2} e^{i \xi}
\end{array}\right)
$$

This potential softly breaks the reflection symmetries $\phi_{1} \rightarrow-\phi_{1}$ and $\phi_{2} \rightarrow-\phi_{2}$. The most general potential of this nature would also have a term $\lambda_{7} \operatorname{Im}\left[\left(\phi_{1}^{\dagger} \phi_{2}\right)^{2}\right]$ but this would induce a non-zero upper component into one of the fields and thereby give rise to an electrically charged vacuum.

At finite temperature and in an electrically neutral plasma we do not anticipate any charge inducing terms in the free energy density (temperature dependent potential) so that a minimum free energy field configuration should also be of the form (7).

Electrically charged fields are not of interest to us in this paper and, as we wish to consider neutral fields not only at a minimum of the free energy but also during the phase transition 
connecting the minimum free energies of the unbroken and broken phases, we take the neutral fields to have the form

$$
\phi_{1}=\left(\begin{array}{c}
0 \\
\rho_{1} e^{-i \theta_{1}}
\end{array}\right) \quad \phi_{2}=\left(\begin{array}{c}
0 \\
\rho_{2} e^{i \theta_{2}}
\end{array}\right)
$$

The Lagrangian density describing the coupling of these Higgs fields with the fermion fields is (using the two component Weyl notation)

$$
\begin{aligned}
\mathcal{L}_{m}= & -\lambda_{u} \rho_{1}\left[u_{r}^{\dagger} e^{-i \theta_{1}} u_{l}+u_{l}^{\dagger} e^{i \theta_{1}} u_{r}\right] \\
& -\lambda_{d} \rho_{2}\left[d_{r}^{\dagger} e^{-i \theta_{2}} d_{l}+d_{l}^{\dagger} e^{i \theta_{2}} d_{r}\right] \\
& -\lambda_{E} \rho_{2}\left[E_{r}^{\dagger} e^{-i \theta_{2}} E_{l}+E_{l}^{\dagger} e^{i \theta_{2}} E_{r}\right]
\end{aligned}
$$

where a summation over generations (and consequently the KM matrix) is implied.

Because of the presence of the interaction term $\operatorname{Im}\left(\phi_{1}^{\dagger} \phi_{2}\right)$ in Eq.(6), away from the false vacuum the Lagrangian is only invariant under changes in the the relative phase $\alpha=\theta_{1}+\theta_{2}$ of the two Higgs doublets, and therefore a small variation in this phase will be shared between $\theta_{1}$ and $\theta_{2}$ according to

$$
d \theta_{1}=\frac{\rho_{2}^{2}}{\rho_{1}^{2}+\rho_{2}^{2}} d \alpha \quad d \theta_{2}=\frac{\rho_{1}^{2}}{\rho_{1}^{2}+\rho_{2}^{2}} d \alpha .
$$

This mode of variation can be shown to be orthogonal to the Goldstone mode which is absorbed into the gauge fields. Thus the partition (10) is the way that change in the phases should be divided even when the fields are not at a free energy minimum, since it is the only remaining physical degree of freedom once the symmetry is broken. In terms of Lorentz covariants we have

$$
\partial_{\mu} \theta_{1}=\frac{\rho_{2}^{2}}{\rho_{1}^{2}+\rho_{2}^{2}} \partial_{\mu} \alpha \quad \partial_{\mu} \theta_{2}=\frac{\rho_{1}^{2}}{\rho_{1}^{2}+\rho_{2}^{2}} \partial_{\mu} \alpha
$$

Following Cohen et al [5], we would like to absorb the phase factors into a redefinition of the fermion fields. In contrast with that work however, we do not have the option of allocating them according to the fermion hypercharge, so that we cannot avoid generating Chern-Simons terms involving the gauge fields. In fact, the relative phases are completely determined by the Higgs potential. Therefore the only remaining freedom is the distribution of the phase absorption between the left and right handed fields. In order to allow for this, we denote a generic Dirac field by $\psi$. The most general rotation

$$
\psi \rightarrow e^{i\left(a+b \gamma_{5}\right) \Theta} \psi
$$

where $a$ and $b$ are constants, generates the additional action term,

$$
\delta S_{0}=-\int \mathrm{d}^{4} x\left[\bar{\psi} m\left(e^{2 i \Theta b \gamma_{5}}-1\right) \psi+\bar{\psi} \gamma^{\mu}\left(a+b \gamma_{5}\right) \psi \partial_{\mu} \Theta\right] .
$$

The axial rotation in the first piece will eventually cancel the complex phase induced by the Higgs potential. The second piece exhibits the conservation or otherwise of the associated current via the Chern-Simons terms. There are two Chern-Simons contributions to the effective potential 
induced by the above rotation. The first type is the anomaly term (the $S U(2)$ pieces coming from the left handed rotations only) which takes the form

$$
\delta S_{1}=\int \mathrm{d}^{4} x\left[i \Theta(a-b) \frac{F_{l} \tilde{F}_{l}}{32 \pi^{2}}-i \Theta(a+b) \frac{F_{r} \tilde{F}_{r}}{32 \pi^{2}}\right]
$$

where $F_{r}\left(F_{l}\right)$ is the gauge field coupling to $\psi_{r}\left(\psi_{l}\right)$. (The coupling constants have been absorbed into $F$ for notational convenience, so that we may include semi-simple Lie groups.) Such terms may be calculated most efficiently using the invariant path integral method as in [19], and are temperature independent as shown in Ref. 20].

In addition to this, the fermion loop diagram in Fig.(1) makes a non-local contribution. In order to determine the effective potential, one makes a Taylor expansion in powers of momenta. This piece then generates a Chern-Simons like term. In calculating this contribution, our approach is analagous to that used in Ref.[2]. That is we consider the fields to be varying slowly enough with time that we may assume a quasi-static equilibrium. This allows us to use the techniques of finite temperature field theory to determine the anomalous contribution to the free energy density. In the calculation of Ref.[2], the time dependence of the field strengths was extracted using the zeroth, (temperature) component of momentum. Therefore the finite temperature anomaly was obtained by making an indirect appeal to the Lorentz invariance of the zero-temperature object from which it came. This procedure is outside the domain of standard finite temperature field theory, and to avoid it, we instead examine the one loop fermion diagrams shown in Fig.(1), and derive the $\Delta$ of Eq.(1) using the space components of the momenta only (for technical details see for example Ref.[21]). The contribution to the action may be expressed as

$$
\delta S_{2}(T)=\int \mathrm{d}^{4} x\left[i b \Theta \Lambda(T)\left(\frac{F_{r} \tilde{F}_{r}}{32 \pi^{2}}+\frac{F_{l} \tilde{F}_{l}}{32 \pi^{2}}\right)\right],
$$

where

$$
\Lambda(T)=\sum \frac{8 \pi T m^{2}}{3\left(m^{2}+(2 n+1)^{2} \pi^{2} T^{2}\right)^{3 / 2}},
$$

and the sum runs over all integer values of $n$. (We remind the reader that the expression above uses the summation over space-time indices merely as a notational convenience, and that finite temperature field theory has no Lorentz invariance.) As pointed out in Ref.[2, 7], there is a complementary topological term generated in the Higgs sector, so that the total topological number $N_{C S}-N_{H}$ is completely gauge invariant. Such terms are generated by the two-loop diagram shown in Fig.(2). At high temperature $T>>m$, we may make the approximation

$$
\Lambda=\frac{14}{3} \zeta(3) \frac{m^{2}}{\pi^{2} T^{2}}\left(1-\frac{93 \zeta(5)}{56 \zeta(3)} \frac{m^{2}}{\pi^{2} T^{2}}+\ldots\right) .
$$

At this point we would like to add a cautionary note regarding thermal calculations of this type. On taking the zero-temperature limit $T \rightarrow 0$, we replace

$$
\sum f((2 n+1) \pi T) \rightarrow \frac{1}{2 \pi T} \int \mathrm{d} \omega f(\omega)
$$

and recover the zero-temperature contribution

$$
\delta S_{2}(0)=\int \mathrm{d}^{4} x\left[i b \Theta\left(\frac{F_{r} \tilde{F}_{r}}{24 \pi^{2}}+\frac{F_{l} \tilde{F}_{l}}{24 \pi^{2}}\right)\right],
$$


which may be recognized as the topological term in Ref.[0]. Alternatively, one may take the zero-mass limit first, upon which we find that $\delta S_{2}(T)=0$ for all temperatures, which seems to contradict Eq.(19). The resolution lies in the fact that in order to make the calculation, we have implicitly assumed that the mass interaction (i.e. the chirality-flip) remains in equilibrium. When taking the $m \rightarrow 0$ limit, at some point this will no longer be the case since (18) is only true for $T<<m$. Thus Eq.(19) will no longer be valid.

On examining the total change in the action, $\delta S=\delta S_{0}+\delta S_{1}+\delta S_{2}(T)$ we find that the charge $(Q)$ and baryon-lepton number $(B-L)$ are conserved at all temperatures, but that $Y$ and $B+L$ are not. Explicitly,

$$
\begin{aligned}
\partial_{\mu} j_{Q}^{\mu} & =0 \\
\partial_{\mu} j_{B-L}^{\mu} & =0 \\
\partial_{\mu} j_{Y}^{\mu}+\ldots & =\left(\frac{3}{4} \Lambda_{b}-\frac{3}{4} \Lambda_{t}+\frac{1}{4} \Lambda_{\tau}\right) \frac{i g_{2}^{2} F_{2} \tilde{F}_{2}}{32 \pi^{2}}+\left(\frac{5}{48} \Lambda_{b}-\frac{17}{48} \Lambda_{t}+\frac{15}{48} \Lambda_{\tau}\right) \frac{i g_{Y}^{2} F_{Y} \tilde{F}_{Y}}{32 \pi^{2}} \\
\partial_{\mu} j_{B+L}^{\mu} & =4 N_{g} \frac{i g_{2}^{2} F_{2} \tilde{F}_{2}}{32 \pi^{2}}-N_{g} \frac{i g_{Y}^{2} F_{Y} \tilde{F}_{Y}}{32 \pi^{2}},
\end{aligned}
$$

where the ellipsis stands for the mass terms in Eq.(13), $F_{2}$ and $F_{Y}$ are the conventional $S U(2)$ and hypercharge field strengths, and $N_{g}$ is the number of fermion generations. Clearly the topological terms in the hypercharge equation would vanish if we were able to take the $T \rightarrow \infty$ limit. Physically what happens is that, as the temperature is increased, the mean free path of particles in the gas becomes much shorter than the path length of the (hypercharge violating) chirality-flip. This is related to our previous point concerning the zero mass limit of $\delta S_{2}$.

We are now ready to apply the method of Ref.[5] to the case under discussion. First we remove the complex phases on the mass terms in Eq.(9) by making the rotations,

$$
\begin{gathered}
u_{r} \rightarrow e^{i\left(\omega-\theta_{1}\right)} u_{r} \quad ; \quad u_{l} \rightarrow e^{i \omega} u_{l} \\
d_{r} \rightarrow e^{i\left(\omega-\theta_{2}\right)} d_{r} \quad ; \quad d_{l} \rightarrow e^{i \omega} d_{l} \\
E_{r} \rightarrow e^{i\left(\bar{\omega}-\theta_{2}\right)} E_{r} \quad ; \quad E_{l} \rightarrow e^{i \bar{\omega}} E_{l},
\end{gathered}
$$

where we have introduced two arbitrary phases, $\omega$ and $\bar{\omega}$. The corresponding changes in the action are,

$$
\begin{aligned}
\delta S_{0}= & -\int \mathrm{d}^{4} x\left[\bar{u}_{l} \gamma^{\mu} u_{l} \partial_{\mu} \omega+\bar{u}_{r} \gamma^{\mu} u_{r} \partial_{\mu}\left(\omega-\theta_{1}\right)+\bar{d}_{l} \gamma^{\mu} d_{l} \partial_{\mu} \omega+\bar{d}_{r} \gamma^{\mu} d_{r} \partial_{\mu}\left(\omega-\theta_{2}\right)\right. \\
& \left.+\bar{E}_{l} \gamma^{\mu} E_{l} \partial_{\mu} \bar{\omega}+\bar{E}_{r} \gamma^{\mu} E_{r} \partial_{\mu}\left(\bar{\omega}-\theta_{2}\right)+\bar{\nu}_{l} \gamma^{\mu} \nu_{l} \partial_{\mu} \bar{\omega}+\ldots\right] .
\end{aligned}
$$

Again the ellipsis refers to the mass terms in Eq.(13). In addition, setting $\Lambda_{b}=\Lambda_{\tau}=0$, we have

$$
\delta S_{1}+\delta S_{2}=\int \mathrm{d}^{4} x\left(18 \omega+6 \bar{\omega}-\frac{3}{2} \Lambda_{t} \theta_{1}\right) \frac{i g_{2}^{2} F_{2} \tilde{F}_{2}}{32 \pi^{2}}-\left(\frac{9}{2} \omega+\frac{3}{2} \bar{\omega}-\left(4-\frac{17}{24} \Lambda_{t}\right) \theta_{1}-4 \theta_{2}\right) \frac{i g_{Y}^{2} F_{Y} \tilde{F}_{Y}}{32 \pi^{2}} .
$$

Instead of solving the equations of motion as in Ref. 2, 7], we may remove the topological piece (i.e. the $S U(2)$ piece) by making a judicious choice of $\omega$ in terms of $\bar{\omega}$, viz.

$$
\omega=\left(\Lambda_{t} \theta_{1}-4 \bar{\omega}\right) / 12 \text {. }
$$

Notice that there is no solution which removes all contributions to Eq.(23), and there remains a term involving the hypercharge fields which is given by,

$$
\delta S_{1}+\delta S_{2}=\int \mathrm{d}^{4} x\left(4 \theta_{2}+\left(4-\frac{13}{12} \Lambda_{t}\right) \theta_{1}\right) \frac{i g_{Y}^{2} F_{Y} \tilde{F}_{Y}}{32 \pi^{2}} .
$$


In the above ,the angles $\omega$ and $\bar{\omega}$ are acting as extra, space dependent $B$ and $L$ rotations. Thus this operation may be interpreted as an absorption of the phase into the right handed fields, followed by the removal of the topological $S U(2)$ piece by an appropriate $B+L$ rotation as given by Eq.20).

Our analysis continues exactly as in Ref. [5]. First we substitute $\omega$ into the current contributions given by (22). We then recalculate the net particle density imposing the above constraints of conserved $B-L$ and $Q$, by including the chemical potentials $\mu_{B-L}$ and $\mu_{Q}$. The relevant expression for the density of a species $i$ is,

$$
\rho_{i}=k_{i}\left(\left\langle\dot{\Theta}_{i}\right\rangle+\left(B_{i}-L_{i}\right) \mu_{B-L}+Q_{i} \mu_{Q}\right) \frac{T^{2}}{6},
$$

where $\Theta_{i}$ is a generic symbol representing the phases appearing in Eq. 222). The factor $k_{i}$ counts colour and spin degrees of freedom, and factors of two for bosons with respect to fermions. This is a relativistic expansion, and there is no Boltzmann suppression, since we are assuming that all species are lighter than $T$ during the phase transition. We now determine $\mu_{Q}$ and $\mu_{B-L}$ by solving $\rho_{Q}=\rho_{B-L}=0$, initially making the naive assumption that all species and sphaleron transitions remain in equilibrium during the phase transition;

$$
\begin{aligned}
\mu_{B-L} & \left.=-\dot{\bar{\omega}}-\left(\left(9-9 \Lambda_{t}+3 n-\Lambda_{t} n\right)\left\langle\dot{\theta}_{1}\right\rangle+24\left\langle\dot{\theta}_{2}\right\rangle\right)\right) /(111+13 n) \\
\mu_{Q} & \left.=-\left(\left(27+\frac{3}{4} \Lambda_{t}\right)\left\langle\dot{\theta}_{1}\right\rangle-\frac{39}{2}\left\langle\dot{\theta}_{2}\right\rangle\right)\right) /(111+13 n) \\
\rho_{B}=\rho_{L} & \left.=\frac{3}{2(111+13 n)}\left(\left(162-51 \Lambda_{t}+18 n-6 \Lambda_{t} n\right)\left\langle\dot{\theta}_{1}\right\rangle+(210+26 n)\left\langle\dot{\theta}_{2}\right\rangle\right)\right) \frac{T^{2}}{6},
\end{aligned}
$$

where $n$ is the number of light charged scalars. As expected, there is no dependence of the final physical quantities, $\rho_{B}$ and $\rho_{L}$, on the arbitrary angle $\bar{\omega}$. Thus the mechanism does not depend on how the phase absorption is divided between left and right handed fields. As in Ref. [5], non-zero phase-changes may bias the sphaleron transitions to produce a net baryon number.

Clearly there are some potential inaccuracies involved in the previous assumptions. The first arises from the fact that the top is the only right handed particle expected to remain in chemical equilibrium during the phase transition. The fact that it is the left-handed particles which contribute to the $S U(2)$ piece of the $\mathrm{B}+\mathrm{L}$ non-conservation, indicates that only the left handed fermions are directly in chemical equilibrium with the sphalerons. For right handed particles to be included, their masses must be sufficiently large that they are able to perform a chirality flip during the phase transition. We should also take account of the fact that there will not be time for the equilibrium densities to be attained. Both these points are dealt with below, where we introduce the relaxation equation which is governed by the sphaleron transition rate. In addition there is the inaccuracy due to the relativistic expansion. Since the major contribution to the baryon asymmetry occurs at the beginning of the phase transition (close to the symmetric phase where the sphaleron transitions are unsuppressed) where the physical top mass is small, this is an acceptable approximation. Because of this it is probably more correct to set $\Lambda_{t} \sim 0$ too.

\section{Comparing the two Approaches}

Our formulation of the Chern-Simons terms in the preceding section allows an easy comparison of the method of Cohen et al applied to this most general case, with that of Turok and Zadrozny 
[2, 7]. In order to give a more reasonable approximation for the former, one should improve on the previous analysis by taking into account the fact that the top quark is the only righthanded particle in equilibrium during the phase transition. Also we use the non-equilibrium rate equation for the relaxation of the particle densities towards their equilibrium values,

$$
\dot{\rho}_{B}=-\Gamma_{B} \mu_{B} / T
$$

$\Gamma_{B}$ is the baryon number violation per unit volume, and is given by

$$
\Gamma_{B}=\kappa \alpha_{W}^{4} T^{4} \mathrm{e}^{-4 \pi V / g_{2} T}
$$

Setting the net densities of the light, right-handed particles to zero, we find that

$$
\mu_{B}=C(n)\left\langle\dot{\theta}_{1}\right\rangle
$$

where

$$
C(n)=-\frac{3}{16} \frac{288-177 \Lambda_{t}+48 n-28 \Lambda_{t} n}{159+25 n},
$$

Thus, making the step-function approximation for $\Gamma_{B}$ as in Ref. [5], we find that

$$
\rho_{B}=C(n) \kappa \alpha_{W}^{4} T^{3} \Delta \theta_{1},
$$

and therefore we can naturally have

$$
\frac{\rho_{B}}{s} \sim 10^{-10}
$$

if $\lambda_{5} \neq \lambda_{6}, \alpha \sim 10^{-2}$, and $\kappa \sim 1$.

In Ref. [2, 8], sphaleron transitions were biased by the one loop diagram contributions using a linearised potential. Instead we may simply absorb the phases in Eq.(9) by rotating the right handed fields only, in order to avoid creating $S U(2)$ anomalies (equivalent to setting $\omega=\bar{\omega}=0$ in Eq.(23)). Thus by Eq.(15) we find

$$
\delta S_{2}=-i \Delta \int \mathrm{d}^{4} x \sqrt{\theta_{1} \theta_{2}} F_{2} \tilde{F}_{2}
$$

where

$$
\Delta=\sum_{i} \frac{7 \zeta(3)}{8 \pi^{2}}\left(\frac{M_{W}^{2}}{\pi^{2} T^{2}}\right) \lambda_{i}^{2}\left(1-93 \zeta(5) / 56 \zeta(3) \frac{m_{i}^{2}}{\pi^{2} T^{2}}+\ldots\right),
$$

where $M_{W}$ is the dynamical quantity, which changes during the phase transition with $\rho_{1}$ and $\rho_{2}$. This reproduces the result of Ref. [2], and inserting the previous numerical values, we obtain

$$
\frac{\rho_{B}}{s}<2 \times 10^{-12}
$$

Clearly, this contribution is suppressed by a factor of $m_{t}^{2} /\left(4 \pi^{2} T^{2}\right) \sim 10^{-2}$ which is the natural parameter of high temperature expansions. Thus one should expect the biasing in this case to be smaller than that caused by the non-zero chemical potentials arising during the phase transition. In addition one should bear in mind that $m_{t}$ (or alternatively the $M_{W}$ appearing in Eq.(35)) is small when the sphaleron transition rate $\Gamma_{B}$ is largest, so that some further suppression may occur. Since in both cases the final baryon asymmetry is proportional to $\alpha$ (assuming that $\rho_{1} / \rho_{2}$ remains relatively constant during the phase transition) it seems that the Turok and Zadrozny effect should be less important as was anticipated in Ref.[5]. 


\section{Supersymmetric Theories}

To conclude we examine the baryon asymmetry for the two supersymmetric theories mentioned in Ref.[5], namely the MSSM and the ESSM. In the first case the superpotential is of the form,

$$
\mathcal{W}=\lambda_{1} Q_{L}^{\dagger} \bar{H}_{1} U_{R}+\lambda_{2} Q_{L}^{\dagger} H_{2} D_{R}+\lambda_{3} L^{\dagger} H_{2} E_{R}+\mu\left(\bar{H}_{1}^{0} H_{2}^{0}-\bar{H}_{1}^{+} H_{2}^{-}\right),
$$

where capital letters implies superfields. After including the soft supersymmetry breaking terms we find that the tree-level neutral Higgs potential is of the form,

$$
\begin{aligned}
V_{0}= & \left(g_{2}^{2}+g_{Y}^{2}\right)\left(\rho_{1}^{2}-\rho_{2}^{2}\right)^{2} / 8+|\mu|^{2}\left(\rho_{1}^{2}+\rho_{2}^{2}\right) \\
& +m_{1}^{2} \rho_{1}^{2}+m_{2}^{2} \rho_{2}^{2}+2|B \mu| \rho_{1} \rho_{2} \cos \left(\theta_{2}-\theta_{1}+\theta_{\mu}+\theta_{B}\right) .
\end{aligned}
$$

Throughout the phase transition, the minimum is given by

$$
\theta_{2}-\theta_{1}=\pi-\theta_{\mu}-\theta_{B}
$$

and therefore for non-trivial values of $\rho_{1}$ and $\rho_{2}$, we must have $\dot{\theta}_{1}=\dot{\theta}_{2}=0$ and so no spontaneous baryogenesis. Alternatively, as in Ref.[22], we could have made a rotation of the Higgs fields to remove the phase on $B \mu$ (that is $\theta_{\mu}+\theta_{B}=0$ ), without affecting the Kobayashi-Maskawa (KM) mixing matrix. We expect that a more full analysis, along the lines of Ref.[6] for the anomaly free (hypercharge coupled) theory, would yield a barely acceptable baryon asymmetry due to one loop corrections to the effective potential. As that work showed, the additional requirements of a sufficiently first order phase transition, and a small neutron electric dipole moment, severely constrain the model.

In the ESSM, the electroweak symmetry breaking is provided by the extra gauge singlet superfield $\Phi$. The superpotential is

$$
\mathcal{W}=\lambda_{1} Q_{L}^{\dagger} \bar{H}_{1} U_{R}+\lambda_{2} Q_{L}^{\dagger} H_{2} D_{R}+\lambda_{3} L^{\dagger} H_{2} E_{R}+\lambda_{7} \Phi\left(\bar{H}_{1}^{0} H_{2}^{0}-\bar{H}_{1}^{+} H_{2}^{-}\right)+\lambda_{8} \Phi^{3} .
$$

Letting the scalar component of the gauge singlet be $\Phi \mid=\rho_{x} e^{i \theta_{x}}$, we find a neutral scalar Higgs potential of

$$
\begin{aligned}
V_{0}= & \left(g_{2}^{2}+g_{Y}^{2}\right)\left(\rho_{1}^{2}-\rho_{2}^{2}\right)^{2} / 8+\left|\lambda_{7}\right|^{2} \rho_{x}^{2}\left(\rho_{1}^{2}+\rho_{2}^{2}\right)+9\left|\lambda_{8}\right|^{2} \rho_{x}^{4}+\left|\lambda_{7}\right|^{2} \rho_{1}^{2} \rho_{2}^{2} \\
& +m_{x}^{2} \rho_{x}^{2}+m_{1}^{2} \rho_{1}^{2}+m_{2}^{2} \rho_{2}^{2}+6\left|\lambda_{7} \lambda_{8}\right| \rho_{1} \rho_{2} \rho_{x}^{2} \cos \left(\theta_{2}-\theta_{1}-2 \theta_{x}+\theta_{\lambda_{7}}-\theta_{\lambda_{8}}\right) \\
& +2\left|A_{7} \lambda_{7}\right| \rho_{x} \rho_{1} \rho_{2} \cos \left(\theta_{2}-\theta_{1}+\theta_{x}+\theta_{\lambda_{7}}+\theta_{A_{7}}\right)+2\left|A_{8} \lambda_{8}\right| \rho_{x}^{3} \cos \left(3 \theta_{x}+\theta_{\lambda_{8}}+\theta_{A_{8}}\right) .
\end{aligned}
$$

We first use the two Higgs rotations to eliminate the phases on the $\lambda_{7}$ and $\lambda_{8}$ couplings. The only physical phases remaining are those on $A_{7}$ and $A_{8}$, the $\theta$ vacuum, and the super-KM and $\mathrm{KM}$ phases [22]. Minimising this potential with respect to phases gives the conditions

$$
\begin{aligned}
& 0=\sin \left(\theta_{2}-\theta_{1}+\theta_{x}+\theta_{A_{7}}\right)+3\left|\frac{\lambda_{8}}{A_{7}}\right| \rho_{x} \sin \left(\theta_{2}-\theta_{1}-2 \theta_{x}\right) \\
& 0=\sin \left(3 \theta_{x}+\theta_{A_{8}}\right)-3\left|\frac{\lambda_{7}}{A_{8}}\right| \frac{\rho_{1} \rho_{2}}{\rho_{x}} \sin \left(\theta_{2}-\theta_{1}-2 \theta_{x}\right) .
\end{aligned}
$$

In the case that $\theta_{A_{7}}-\theta_{A_{8}}=\pi$ we have the trivial minimisation (i.e. when all the cosines in Eq.(41) are -1 ) given by

$$
\begin{aligned}
\theta_{2}-\theta_{1} & =\pi-\frac{2}{3} \theta_{A_{7}} \\
\theta_{x} & =-\frac{1}{3} \theta_{A_{7}}
\end{aligned}
$$


in which case there is again no spontaneous baryogenesis, since the $A_{7}$ coupling is approximately constant during the phase transition. (We make this last assumption because the phase transition is taken to be first order, in which case it proceeds by spinodal decomposition implying that the temperature remains nearly constant.) Now let $\theta_{A_{7}}-\theta_{A_{8}}=\pi+\Delta \theta_{A}$, where we assume that $\Delta \theta_{A}$ is small. Then the minimum may be approximated by

$$
\begin{aligned}
\theta_{2}-\theta_{1} & =\pi-\frac{2}{3} \theta_{A_{7}}-\Delta \theta_{A} \frac{A_{8} \rho_{x}\left(6 \lambda_{8} \rho_{x}-A_{7}\right)}{3\left(3 A_{7} \lambda_{7} \rho_{1} \rho_{2}+A_{7} A_{8} \rho_{x}+3 A_{8} \lambda_{8} \rho_{x}^{2}\right)} \\
\theta_{x} & =-\frac{1}{3} \theta_{A_{7}}-\Delta \theta_{A} \frac{A_{8} \rho_{x}\left(3 \lambda_{8} \rho_{x}+A_{7}\right)}{3\left(3 A_{7} \lambda_{7} \rho_{1} \rho_{2}+A_{7} A_{8} \rho_{x}+3 A_{8} \lambda_{8} \rho_{x}^{2}\right)} .
\end{aligned}
$$

It should be noted that the residual angle appearing in the mass terms may be absorbed by fermion rotations without affecting the KM matrix, exactly as in the MSSM. If we again make the assumption that the vacuum expectation values are small when the baryon number violation is greatest, we may approximate $\theta_{2}-\theta_{1}=\pi-\left(2 \theta_{A_{7}}-\Delta \theta_{A}\right) / 3$, again implying no baryon asymmetry. Clearly the production of a net baryon number depends on how long the sphaleron transitions are allowed to continue away from the symmetric phase. In order to make an estimate, we shall use the renormalisation results of Ref. [23], for the supergravity inspired model with the parameters (in the notation of that paper), $m_{1 / 2}=200 \mathrm{GeV}, m_{0}=300 \mathrm{GeV}, \tan \beta=2, \tan \beta_{x}=1 / 2$. In addition we use the values for the couplings $\lambda_{7}=g_{2}, \lambda_{8}=0.2$. We may induce a nonzero $\Delta \theta_{A}$ by allowing a small phase on the trilinear (soft-supersymmetry breaking) parameter $A=|A| \exp i \Delta \theta_{A_{0}}$. We shall choose $|A|=0.2$.

Close to the GUT scale $\Delta \theta_{A}=0$, but as the renormalisation group equations are run down to the weak scale, a non-zero phase develops. At the beginning of the phase transition since the phases on $A_{7}$ and $A_{8}$ are constant, there is no baryon number production. Towards the end of the phase transition however, the phases are changing rapidly due to the large vacuum expectation values that the Higgs fields have aquired. The total change in phases is

$$
\Delta \theta_{2}-\Delta \theta_{1}=3.3 \Delta \theta_{A_{0}}
$$

and hence,

$$
\frac{\rho_{B}}{s} \sim 10^{-8} \Delta \theta_{A_{0}}
$$

In this case, the observed baryon asymmetry may quite easily be generated, provided that sphaleron transitions are not frozen out too quickly. In addition, we note from Ref. 177 that the phase transition may be naturally first order, since there are trilinear terms in the Higgs potential even at tree level. This also implies that there may be some enhancement of the baryon asymmetry arising from transport processes as described in Ref.[0].

Acknowledgement We would like to thank L. McLerran for useful discussions, and S. Sarkar for a critical reading of the manuscript. Two of us [SAA,WNC] would like to thank James Cook University for hospitality extended during the completion of this work. 


\section{References}

[1] A. G. Cohen and D. B.Kaplan, Phys. Lett. B199 (1988) 251

[2] N. Turok and J. Zadrozny, Phys. Rev. Lett. 65 (1990) 1

[3] A. I. Bochkarev, S. V. Kuzmin and M. E. Shaposhnikov, Phys. Lett. B244 (1990) 275

[4] L. McLerran, M. Shaposhnikov, N. Turok and M. Voloshin, Phys. Lett. B256 (1991) 451

[5] A. G. Cohen, D. B.Kaplan and A. E. Nelson, Phys. Lett. B263 (1991) 86; Nuc. Phys. B373 (1992) 453

[6] A. G. Cohen and A. E. Nelson, Boston Preprint BU-HEP-92-20 (1992)

[7] N. Turok and J. Zadrozny, Nuc. Phys. B358 (1991) 471

[8] N. Turok and J. Zadrozny, Nuc. Phys. B369 (1992) 729

[9] D. Grigoriev, M. Shaposhnikov and N. Turok, Phys. Lett. B275 (1992) 395

[10] M. Dine, P. Huet and R. Singleton, Nuc. Phys. B375 (1992) 625

G. F. Giudice, Phys. Rev. D45 (1992) 3177

[11] S. Myint, Phys. Lett. 287B (1992) 325

[12] A. Linde, Stanford Preprint SU-ITP-92-18 (1992)

M. E. Shaposhnikov, Cern Preprint CERN-TH 6497/92 (1992)

M. Dine, Santa Cruz Preprint SCIPP 92/21 (1992)

[13] G. 't Hooft, Phys. Rev. Lett. 37 (1976) 8

[14] M. E. Shaposhnikov, Nuc. Phys. B287 (1987) 757; Nuc. Phys. B (1988) 797

[15] A. D. Sakharov, Pisma Zh. Eksp. Teor. Fiz. 5 (1967) 32; (JETP Lett. 5 (1967) 24)

[16] D. A. Kirzhnits and A. D. Linde, Ann. Phys. 101 (1976) 195

A. D. Linde, Phys. Lett. 70B (1977) 306; Nuc. Phys. B216 (1983) 421

V. A. Kuzmin, V. A. Rubakov and M. E. Shaposhnikov, Phys. Lett. 155B (1985) 36

D. Brahm and S. Hsu, CALT 68-1705; CALT 68-1762

G. Anderson and L. Hall, LBL-31169 (1991)

M. Dine, R. G. Leigh, P. Huet, A. Linde and D. Linde, Phys. Lett. 283B (1992) 319

[17] M. Pietroni, Padova Preprint DFPD/92/TH/36 (1992)

[18] J. F. Gunion, H. E. Haber, G. L. Kane and S. Dawson," The Higgs hunter's guide" (AddisonWesley, Reading, MA 1990)

[19] K. Fujikawa, Phys. Rev. Lett. 42 (1979) 1195; Phys. Rev. D21 (1980) 2848

[20] M. Reuter and W. Dittrich, Phys. Rev. D32 (1985) 513

[21] J. I. Kapusta, "Finite Temperature Field Theory" (CUP, 1989) 
[22] M. J. Duncan, Nuc. Phys. B221 (1983) 285

M. Dugan, B. Grinstein and L. J. Hall, Nuc. Phys. B255 (1985) 413

[23] S. A. Abel, S. Sarkar and I. B. Whittingham, Oxford Preprint OUTP-92-10P (1992) 


\section{Figures}

Figure 1 The triangle diagram leading to anomalous Chern-Simons terms in the effective potential, and also to finite temperature non-anomalous contributions.

Figure 2 The Higgs equivalent to Fig.(1), which leads to a Higgs topological term, $N_{H}$. This ensures that the quantity $N_{C S}-N_{H}$ is total gauge invariant which may be described as the total derivative of a density, the Goldstone-Wilczek density. 